\title{
Evidence of a Low-Mass Companion to AB Doradus
}

J. C. Guirado ${ }^{1,2}$, J. E. Reynolds ${ }^{3}$, J.-F. Lestrade ${ }^{4}$, R. A. Preston ${ }^{1}$, D. L. Jauncey ${ }^{3}$, D. L. Jones ${ }^{1}$, A. K. Tzioumis ${ }^{3}$, R. H. Ferris ${ }^{3}$, E. A. King ${ }^{5}$, J. E. J. Lovell ${ }^{5}$, P. M. McCulloch ${ }^{5}$, K. J. Johnston ${ }^{6}$, K. A. Kingham ${ }^{6}$, J. O. Martin ${ }^{6}$, G. L. White ${ }^{7}$, P. A. Jones ${ }^{7}$, F. Arenou ${ }^{4}$, M. Froeschlé ${ }^{8}$, J. Kovalevsky ${ }^{8}$, C. Martin ${ }^{8}$, L. Lindegren ${ }^{9}, \&$ S. Soderhjelm ${ }^{9}$

Abstract. From the combination of VLBI phase-referenced observations and Hipparcos satellite data, we have found evidence of a low-mass object orbiting the late-type star AB Doradus. The mass of the new object is near the hydrogen burning limit and will constitute a precise point for calibrating the low end of the main sequence. This represents the first detection of a low-mass stellar companion using the VLBI technique, which could become an important tool in future searches for planets and brown dwarfs orbiting other stars.

\section{Introduction}

Determination of star's kinematics with submilliarcsecond precision provides not only the position, proper motion, and parallax of the observed star but possible accelerations caused by the gravitational interaction with companion objects. Unlike radial velocity techniques, astrometry-based techniques, such as VLBI or Hipparcos satellite data, are able to determine a full set of orbital elements which provides an unambiguous estimate of the mass of the companion.

\section{Observations and Results}

We have combined two astrometric data types, VLBI phase-referenced data and Hipparcos satellite data, to monitor the position of the Southern K0-star AB Doradus (=HD $36705=\mathrm{HIP} 25647$, AB Dor hereafter). The $8.4 \mathrm{GHz}$ VLBI data were originally intended to contribute to the determination of the link between the Hipparcos and VLBI reference frames (Lindegren \& Kovalevsky 1995). The VLBI array was composed of the Australian antennas at Tidbinbilla, Parkes, and Hobart; the data were correlated at the MkIIIA correlator of the US Naval Observatory at Washington, DC. We followed the astrometric VLBI technique described in Lestrade et al. (1990) to estimate the star's coordinates, referred to the IERS reference frame. The coordinates from each data type, VLBI and Hipparcos satellite, showed independent evidence of a previously unseen non-linear motion on the sky of AB Dor, indicating that this star was likely an astrometric double. Since the final Hipparcos catalogue (ESA 1997) is also linked to the IERS reference system (Kovalevsky et al. 1997), we could use the joint VLBI

\footnotetext{
${ }^{1}$ Jet Propulsion Laboratory, California Institute of Technology, Pasadena, CA 91109, USA; ${ }^{2}$ Departamento de Astronomía, Universitat de València, 46100 Burjassot, València, Spain; ${ }^{3}$ Australian Telescope National Facility, Epping, New South Wales 2121, Australia; ${ }^{4}$ Observatoire de Paris-Meudon-CNRS, F-92195 Meudon Principal Cedex, France; ${ }^{5}$ University of Tasmania, Hobart, Tasmania 7001, Australia; ${ }^{6}$ U.S. Naval Observatory, Washington D.C., 20392, USA; ${ }^{7}$ University of Western Sydney, Sydney, New South Wales, Australia; ${ }^{8}$ Observatoire de la Côte d'Azur, CERGA, F-06130 Grasse, France; ${ }^{9}$ Lund Observatory, S22100 Lund, Sweden
} 


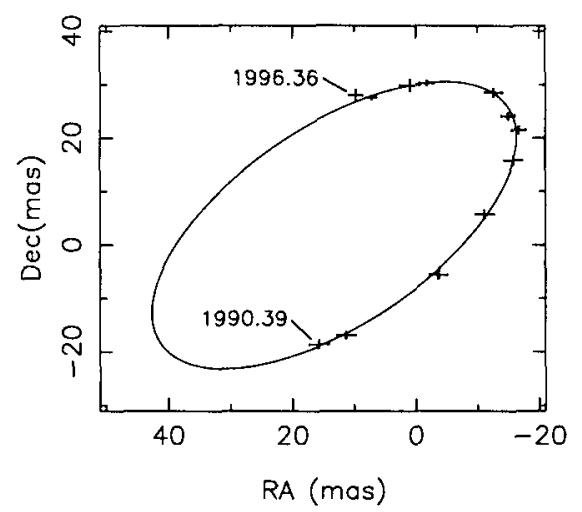

Figure 1. The apparent orbit for the reflex motion of $A B$ Dor corresponding to a companion mass of $0.094 \mathrm{M}_{\odot}$. The five earliest epochs correspond to Hipparcos data. The continuous line represents the least-square fitted reflex orbital motion of AB Dor.

+ Hipparcos data set to estimate simultaneously the position, proper motion, parallax, and orbital elements of the reflex motion of $\mathrm{AB}$ Dor. Although the combined data did not cover a full orbit, we investigated the astrometric and orbital parameter space to find orbits compatible with our positions (see Guirado et al. 1997 for a detailed analysis). The main results of our search are: i) a new value of the parallax of $\mathrm{AB}$ Dor $(66.3<\pi<67.2$ milliarcseconds), which, in turn, provided a revised mass for $\mathrm{AB}$ Dor, $0.76 \mathrm{M}_{\odot}$, and $\left.i i\right)$ the dynamical mass of the unseen companion, which is constrained to lie between 0.08 and $0.11 \mathrm{M}_{\odot}$ (see Fig 1).

The newly discovered companion, AB Dor C, is one of the few low mass objects near the hydrogen burning limit whose mass has been determined dynamically (Henry \& McCarthy 1993). A suitable photometric calibration would locate this object in a mass-luminosity diagram and would constitute a new precise point for the calibration of the low end of the main sequence (e.g., Baraffe et al. 1995). The detection of AB Dor $C$ demonstrates the capability of VLBI phase-referencing, enhanced in this case with Hipparcos data, for detecting very low-mass companions orbiting stars.

Acknowledgments. This research was performed while J.C.G. held a NRC-NASA Resident Research Associateship award at JPL, California Institute of Technology. Research at JPL is carried out under contract with the NASA. The ATNF is funded by the Commonwealth Government as a national facility managed by CSIRO. The optical contribution to this research is based on observations made with the ESA Hipparcos satellite.

\section{References}

Baraffe, L., et al. 1996. ApJ, 446, L35-38.

ESA, 1997. The Hipparcos and Tycho catalogues, ESA SP-1200.

Guirado, J. C., et al. 1997. $A p J, 490,835-839$.

Henry, T. J. \& McCarthy, D. W. 1993. AJ, 106, 773-789.

Kovalevsky, J., et al. 1997. $A \& A, \mathbf{3 2 3}, 620-633$.

Lestrade, J.-F. et al. 1990. $A J, 99,1663-1673$.

Lindegren, L. \& Kovalevsky, J. 1995. A\&A, 304, 189-201. 